Regular article

\title{
Rapid formation of biofilm grown on gas-permeable membrane induced by famine incubation
}

\author{
Tinggang $\mathrm{Li}^{\mathrm{a}, *, 1}$, Junxin $\mathrm{Liu}^{\mathrm{a}, \mathrm{b}, *}$ \\ ${ }^{a}$ Research Center for Eco-Environmental Sciences, Chinese Academy of Sciences, Beijing 100085, People's Republic of China \\ ${ }^{\mathrm{b}}$ University of Chinese Academy of Sciences, Beijing 100049, People's Republic of China
}

\section{A R T I C L E I N F O}

\section{Article history:}

Received 2 November 2016

Received in revised form 17 January 2017

Accepted 21 January 2017

Available online 3 February 2017

\section{Keywords:}

Biofilm

Famine incubation

Biofilm formation rate

Extracellular polymeric substances

Surface charge

Hydrophobicity

\begin{abstract}
A B S T R A C T
A new approach to rapidly initiate biofilm induced by famine incubation was developed in a membraneaerated biofilm reactor (MABR). The correlation between extracellular polymeric substances (EPS) component, surface physiochemical properties of biofilm, and biofilm formation rate was evaluated. The results indicated that famine incubation appeared to stimulate bacteria to secrete more bound EPS and resulted in improvement in surface properties of cells in the culture favoring the accumulation of biofilm flocs onto the membrane surface. High contents of bound EPS and bound protein along with the reduced surface charges enhanced hydrophobicity of bacteria. Biofilm formation rate strongly correlated with EPS component and bindability rather than the concentration of total EPS. The study suggested that famine incubation may provide an alternative tool to initiate the rapid formation of biofilm by regulating the component and surface characteristics of biofilm-EPS matrix, which enhanced the efficacy of membrane-aerated biofilm (MAB) for high-rate organic carbonaceous pollutant biodegradation, volatile organic pollutant removal, and nitrification/denitrification processes.
\end{abstract}

(c) 2017 Elsevier B.V. All rights reserved.

\section{Introduction}

Biofilms form at surface-associated interfaces and consist of a hydrated mix of bacterial cells, their extracellular polymeric substance (EPS) and particulate matter [1,2]. Biofilm presents several advantages to the bacteria and other microorganisms over a planktonic mode of existence, such as increased resistance to environmental stresses [3], remarkable survival mechanisms [4], potential for horizontal gene transfer, and adaptation to the presence of xenobiotics in their environment $[5,6]$. In general, the transition from free-living cells to a sessile form of life begins with the attachment, followed by development of biofilm flocs onto a substratum. The heterogeneous microenvironment of living cells in microbial biofilms is mediated by an EPS matrix which produces changes in biofilm porosity, density, surface charge, viscosity, hydrophobicity, and mechanical stability [7,8]. The physico-chemical characteristics of the surface may in turn influence the interaction between microbial aggregates.

\footnotetext{
* Corresponding authors. Tel.: +86 10 62849133; fax: +86 1062849133

E-mail addresses: tglibj@hotmail.com (T. Li), jxliu@rcees.ac.cn (J. Liu).

1 Present address: Department of Civil and Environmental Engineering, National University of Singapore.
}

Bacterial cell surface hydrophobicity can affect bacterial adhesion to different types of substrates [9]. Surface charge is suggested to play an important part in the early formation of biofilms [10]. However, the biofilm formation is a complicated process involving both biological and physicochemical factors. Not all cultures successfully attach and grow to form biofilms on gas-permeable membranes, especially for a membrane-aerated biofilm (MAB) [11].

The membrane-aerated biofilm reactor (MABR) has emerged as a promising alternative technology that can achieve bubbleless aeration and high oxygen utilization efficiency (up to 100\%), thereby enhancing the biofilm activity. In the MABR, oxygen and substrates were supplied to the biofilm from opposite sides, in contrast to those from the same side in conventional biofilm bioreactors. The biofilm can be stratified into aerobic/anoxic/anaerobic zones to simultaneously achieve removal of carbonaceous organic pollutants as well as nitrification and denitrification (if needed) in a single biofilm $[12,13]$. The development of efficient strategies to enhance the biofilm formation requires a more thorough understanding of formation behavior of biofilms, thereby to some extent shortening the time of colonization during the start-up phase, mitigating the need for carrier surface condition and/or modification for biofilm attachment $[14,15]$, and efforts to immobilize the desired bacteria (e.g. nitrifying bacteria and volatile organic compounds degrading bacteria) in an MABR for resistance to environmental stresses [12]. 
Although natural biofilm formation has been extensively studied, it is not economically feasible in large scale technologies because of the length of time required to achieve the desired amount of immobilised biomass per unit volume. This issue has driven research into alternative methods of rapid biofilm formation, specially the impact of EPS component and characteristics (solubility and bindability), and physiochemical properties on biofilm formation rate under varying operational conditions (e.g. surface loading rate (SLR) and liquid recirculation rate). Therefore, the overall objective of this study was to develop an approach to enhance microbial biofilm formation in an MABR. Factors affecting biofilm formation rate such as EPS quantity, component, characteristics, and surface physicochemical properties of biofilm biomass were examined under the famine incubation at a low SLR. This work provides the insight into conditions strengthening or weakening microbial biofilm formation and sloughing, which will benefit to improve the design and operation of such systems for future applications of biofilm technology.

\section{Materials and methods}

\subsection{Gas-permeable membrane and reactor}

A gas-permeable membrane was made of polypropylene ( $320 \mu \mathrm{m}$ o.d. and $200 \mu \mathrm{m}$ i.d.) (Zenon, Singapore). The gaspermeable membrane had surface zeta potential of $-39.3 \pm 0.4 \mathrm{mV}$ $(\mathrm{pH}=7)$ and membrane surface hydrophobicity with contact angle of $108.9 \pm 1.4^{\circ}$. An aeration membrane module with a specific membrane surface area of $84.5 \mathrm{~m}^{2} / \mathrm{m}^{3}$ in a dead-end configuration was placed in an enclosed pyrex cylinder (a working volume of $1.54 \mathrm{~L}$ ) as previously described [16].

\subsection{Experimental approach of biofilm formation}

Two series of runs were conducted to assess the effect of famine incubation on formation rate of biofilm, variations of biofilm physiochemical properties, and the mechanisms of biofilm formation. A gas-permeable membrane module was submerged in an MABR to support biofilm growth over a 15-day period. Two reactors were performed under the same experimental procedures with different SLRs. Firstly, for the famine incubation run at a low SLR of $3.4 \mathrm{~g} /\left(\mathrm{m}^{2} \mathrm{~d}\right)(0.27 \mathrm{~g}$-acetonitrile/L of influent), the reactor was first seeded with acclimatized sludge with an initial volatile suspended solid (VSS) concentration of $2000 \mathrm{mg} / \mathrm{L}$. Following inoculation, the reactor was operated in a continuous mode for 2 days, the reactor operation was then stopped and all the suspended biomass in the reactor were removed. The reactor was then subjected to the famine incubation fed with acetonitrile for a period of 13 days. For the control run, reactor culture was subjected to a high SLR of $12.8 \mathrm{~g} /\left(\mathrm{m}^{2} \mathrm{~d}\right)$ (1.02 g-acetonitrile/L of influent) under the identical experimental approach as compared to the famine incubation run. In each run, bubbleless aeration was provided at a flowrate of 13.1-16.8 $\mathrm{mL}^{-} \mathrm{O}_{2} / \mathrm{min}$ at a trans-membrane oxygen partial pressure of 20.7-27.6 kPa to maintain the dissolved oxygen (DO) concentration at above $1.5 \mathrm{mg} / \mathrm{L}$ in the bulk liquid. Centrifugal pumps were used to recirculate the liquid in the reactor at a rate of $0.9 \mathrm{~L} / \mathrm{min}$ to achieve a laminar flow region $(\mathrm{Re}=1527)$ with a mean fluid velocity of $3 \mathrm{~cm} / \mathrm{s}$ in the reactor. The reactor was operated at $25 \pm 1^{\circ} \mathrm{C}$ in a temperature controlled room. Biofilms were harvested from gas-permeable membrane modules for further examination at intervals.

\subsection{Biofilm formation rate}

Biofilm formation rates were measured under the famine incubation run and the control run, respectively. These activity tests for biofilm formation were based on measuring the amount of deposited bioflim biomass per unit of time, normalized by the membrane area and, expressed in $\mathrm{mg}-\mathrm{VSS} /\left(\mathrm{m}^{2} \mathrm{~d}\right)$. For each reactor, the biofilm formation rate coefficient, $k(\mathrm{~m} / \mathrm{s})$, was calculated as the ratio between the biofilm formation rate and the initial concentration (expressed in $\mathrm{mg}-\mathrm{VSS} / \mathrm{m}^{2}$ ). Therefore, the biofilm rate coefficient, $k$, was defined as:

$V \frac{\mathrm{d} C}{\mathrm{~d} t}=-k A C$

where $k$ can be derived as follows under an initial condition of $C=C_{0}$ at $t=0$ :

$k=-\left(\frac{\mathrm{V}}{A}\right)\left(\frac{1}{t}\right) \ln \left(\frac{C_{t}}{C_{0}}\right)$

Where $V, A, t, C_{\mathrm{t}}$, and $C_{0}$ are the working volume of the MABR, membrane surface area, contact time, attached biofilm biomass concentration at time $t$ and the initial concentration at $\mathrm{t}=0$. All biofilm formation rate coefficients are averages taken from three experiments conducted under the same experimental conditions.

\subsection{Sampling and general analysis}

Biofilms were harvested from the gas-permeable membrane test module in the reactor grown over a 15-day period under famine incubation. Samples attached to the gas-permeable membrane were excised with a razor blade and each segment was placed in a sterile centrifuge tube and stored at $-20^{\circ} \mathrm{C}$ for further analysis. Mixed liquor suspended solid (MLSS) and volatile suspended solid (VSS) were measured according to standard methods [17]. Acetonitrile and its biodegradation intermediates were measured through gas chromatography (Agilent 6890N, USA) as previously described [18]. Biofilm thickness was measured optically using a traveling micromanipulator microscope with in the $x, y$ and $z$ directions (Keyence,Japan). Biofilm formation morphology was observed with a scanning electron microscope (SEM) (JSM-5310LV, Tokyo, Japan) after the fixation, dehydration and $\mathrm{CO}_{2}$ critical drying of biofilm samples.

\subsection{EPS extraction and quantitative analysis}

EPS were characterized in terms of soluble or bound polysaccharide and protein. EPS extraction was carried out according to the previous extraction protocol [16]. Briefly, the biofilm biomass from each slice was prepared into a $5 \mathrm{~mL}$ suspension with the addition of sterile deionized water. Soluble EPS content (soluble polysaccharide and soluble protein) in the supernatant was physically extracted, without adding any chemical extractant, whereas bound EPS content (bound polysaccharide and bound protein) attached the biofilm floc was chemically extracted using formaldehyde plus $\mathrm{NaOH}$ extractant. The EPS was analyzed for total polysaccharide and protein, which are the dominant components typically found in extracted EPS [19]. Polysaccharide concentrations were quantified by the phenol-sulfuric acid method with glucose as the standard [20]. Protein concentrations were determined using the Bradford coomassie Blue method with Bovine Serum Albumin (BSA) as the standard [21]. All samples were analyzed using a UV-vis spectrophotometer (Jasco V-550, Japan) at the wavelength of 490 and $595 \mathrm{~nm}$ for polysaccharide and protein, respectively. The total EPS were defined as the sum of the soluble EPS and the bound EPS.

\subsection{Surface charge analysis}

Cell surface charge was expressed as zeta potential. Biofilm mass samples were dispersed by vortexing them over four cycles of $15 \mathrm{~s}$ on and $15 \mathrm{~s}$ off. The concentration of wet biomass was maintained at 


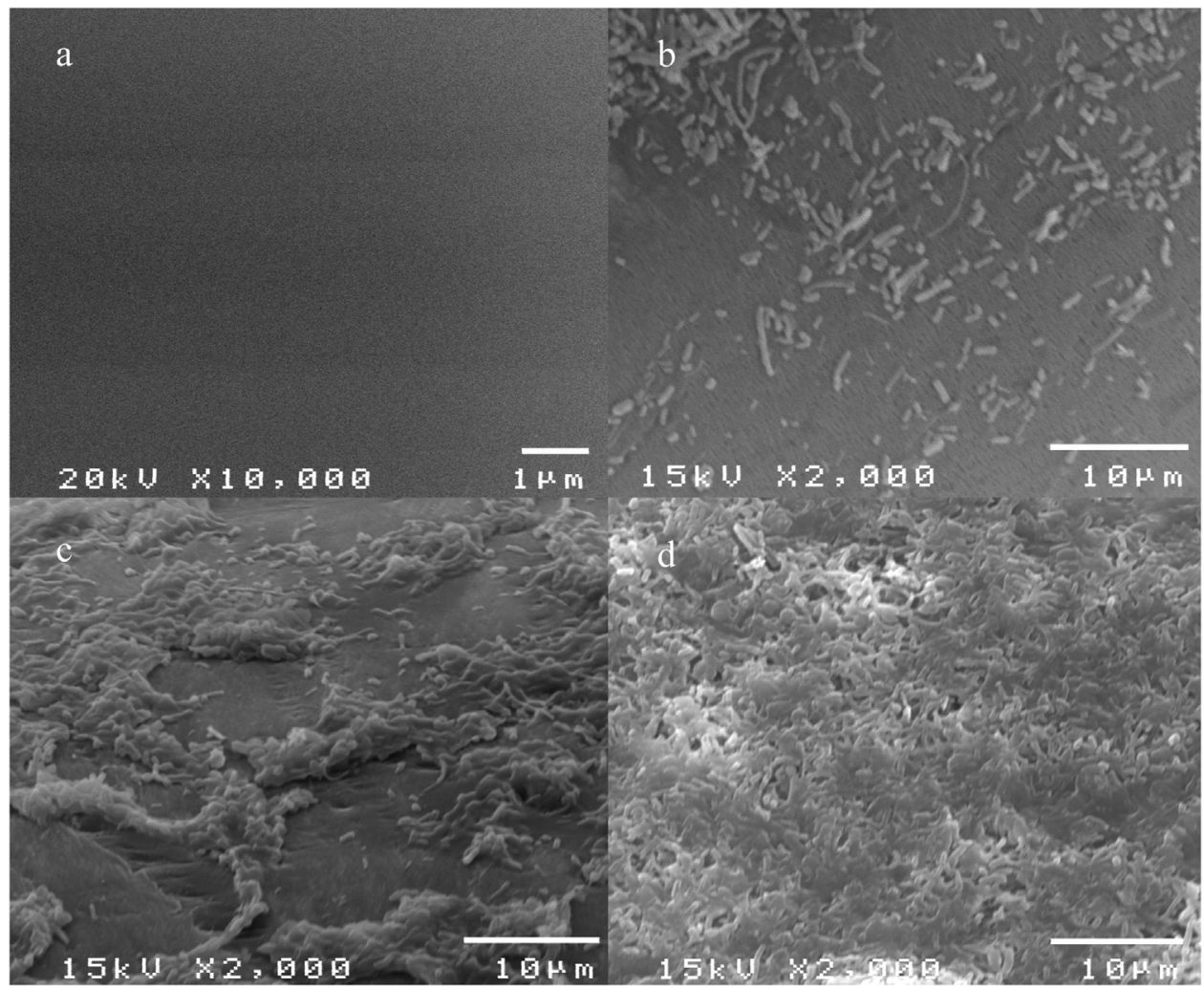

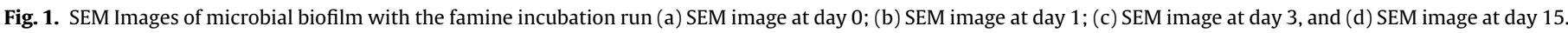

about $0.02 \%$ on a wet-weight basis via dilution with Mili-Q water and used for the measurement of zeta potential. Zeta potentials were measured by a Zeta Potential Analyzer (ZetaPals, Brookhaven Instruments, Holtsville, NY) and analyzed by a software of BIC Pals Potential analyzer.

\subsection{Hydrophobicity analysis}

Hydrophobicity of biofilm biomass was carried out by water contact angle measurement using a contact angle system (OCA 20, Dataphysics Instruments Gmbh, Germany). The biofilm mass sample was transferred onto a cellulosic membrane held on a glass filter. Under even suction filtration, the biofilm mass was deposited on the cellulosic membrane surface, then carefully transferred onto the surface of a freshly prepared $2 \%$ solidified agar plate to preserve the moisture content of the cake. After $4 \mathrm{~h}$ moisture equilibration between the agar and biofilm mass cake, the agar plate was then placed in the contact angle system. A drop of double distilled deionized water was placed on the biofilm mass cake using a Gilmont micrometer syringe equipped with a stainless steel needle. A video image system was used to view the sessile drop from the top. The drop shape was captured after no further shrinking the water drop was observed. The image of sessile drops was used to determine contact angle values.

\subsection{Viscosity analysis}

The apparent viscosity was determined using a rotational viscometer (Model LVDVII, Brookfield Viscometers, UK). Due to the non-Newtonian nature of the biomass flow, the shear stress was non-linearly related to the shear rate. The apparent viscosity, expressed as mPa s, was quoted at a shear rate of $22 \mathrm{~s}^{-1}$.

\subsection{Statistical analysis}

Statistical analyses were performed to evaluate the major factors affecting biofilm formation rate through univariate linear correlation analysis. Although many relations are probably not linear, this analysis by means of linear correlations can provide an initial estimate of the strength of the correlations and thus can be used to identify the major correlations. The statistical analysis was carried out with Statistical Package for the Social Sciences (SPSS). The Pearson's product momentum correlation $\left(r_{\mathrm{p}}\right)$ was used for linear estimations of the strength and direction of linear correlations between every two parameters. The Pearson's $r_{\mathrm{p}}$ coefficient can take on the values in the range of -1 and +1 , where $r_{p}$ close to -1 or +1 means a good positive or negative correlation, 0 means an absence of a relationship. In this study, correlations were considered statistically significance at a $95 \%$ confidence interval $(p<0.05)$.

\section{Results}

\subsection{Morphological observation and evolution characteristics of biofilms}

The MABR culture was subjected to the famine incubation under the low SLR of $3.4 \mathrm{~g}-\mathrm{ACN} /\left(\mathrm{m}^{2} \mathrm{~d}\right)$. Biofilm formation was expressed qualitatively by SEM observation (Fig. 1a-d). The microbial attachment to the gas-permeable membrane surface took place immediately after famine incubation for day 1 . Microbial cells dispersedly attached to the surface of the gas-permeable membrane (Fig. 1b). To avoid biomass detachment at the initial stages of biofilm development, a relatively low upflow recirculation rate at $3 \mathrm{~cm} / \mathrm{s}$ was applied to the MABR. After 3 days of continuous incubation, EPS containing adhesive structures appeared around microbial cells favoring their aggregation on the gas-permeable membrane surface. However, carrier membrane surfaces had irregular and 


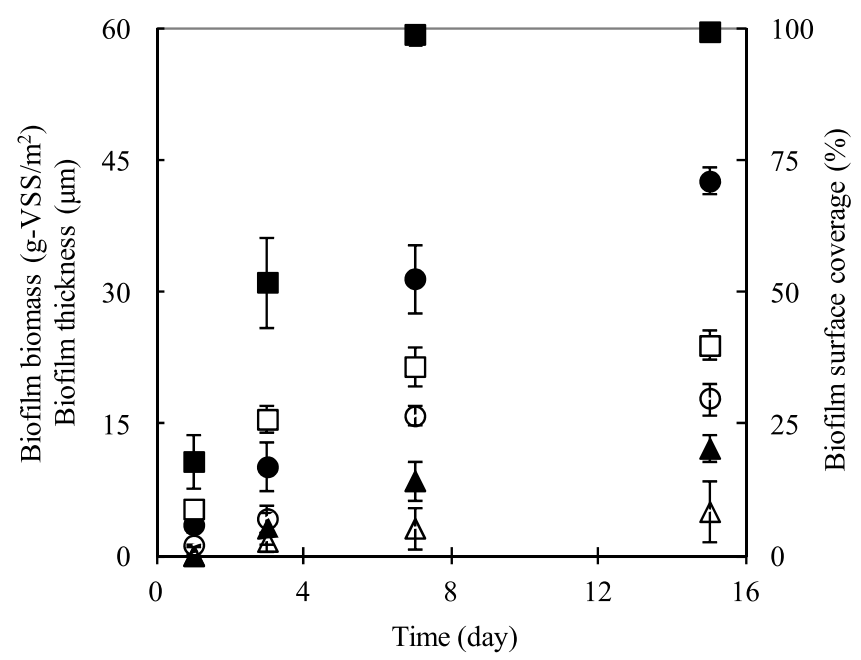

Fig. 2. Evolution of biofilm formed in an MABR as a function of incubation time under the famine incubation run ( $\boldsymbol{\Lambda}$, biofilm biomass; $\bullet$, biofilm thickness; $\mathbf{\square}$, biofilm surface coverage) and the control run ( $\Delta$, biofilm biomass; $\bigcirc$, biofilm thickness; $\square$, biofilm surface coverage). Vertical bars represent the standard deviations calculated from triplicates.

uneven patches indicative of heterogeneous formation of biofilm on gas-permeable membrane surfaces (Fig. 1c). A thin biofilm was visually covered by the membrane surface after approximately 7-15 days of operation (Fig. 1d). It was observed that the biofilms were composed of rod-shaped bacteria surrounded with a large amount of EPS.

Fig. 2 depicts the quantitative evolution characteristics of microbial biofilms as a function of operational time in the first 15 days. The attached biofilm biomass, biofilm thickness and biofilm surface coverage exhibited similar variation patterns within the incubation time. For the famine incubation run, the biomass immobilized on the membrane and biofilm thickness increased throughout the experiment from 0.13 to $3.2 \mathrm{~g}-\mathrm{VSS} / \mathrm{m}^{2}$ and 3.6-10.2 $\mu \mathrm{m}$ in the first 3 days, and from 8.6 to $12.3 \mathrm{~g}-\mathrm{VSS} / \mathrm{m}^{2}$ and $31.6-42.7 \mu \mathrm{m}$ for the rest of over two thirds of the incubation time. In contrast to the control run, the biofilm formation was rapidly initiated with about 2 times increase in biofilm surface coverage on day 3 as biofilm flocs occurred in the famine incubation run (see Fig. 2).

Biofilm formation rate coefficient was examined to determine the corresponding changes in biofilm biomass per unit time (Fig. 3). For the control run, biofilm formation rate coefficients were insignificant during the first 15 days due to the small amount of the attached biofilm mass on the membrane surface. However, the biofilm formation rate coefficient showed an rapid upward trend from day 1 onwards, and enhanced over 1.8 times with the highest value $\left(7.76 \pm 0.45 \times 10^{-6} \mathrm{~m} / \mathrm{s}\right)$ on day 15 in the famine incubation run as compared to that in the control run (Fig. 3). The biofilm eventually grew to attach to the surface of gas-permeable membrane after approximately 7-15 days of operation, as evidenced by the gradual increase in the mean biofilm thickness and biofilm surface coverage under the famine incubation run (Fig. 2). Consequently, the adapted famine incubation strategy presented in this study has greater potential for formation of microbial biofilm grown on the gas-permeable membrane from the practical application and research point of view.

\subsection{Triggering forces and correlations of biofilm formation under famine incubation}

To better understand the interactions between biofilm formation rate and formation factors involved in famine-induced biofilm formation, further examination of EPS components and characteris-

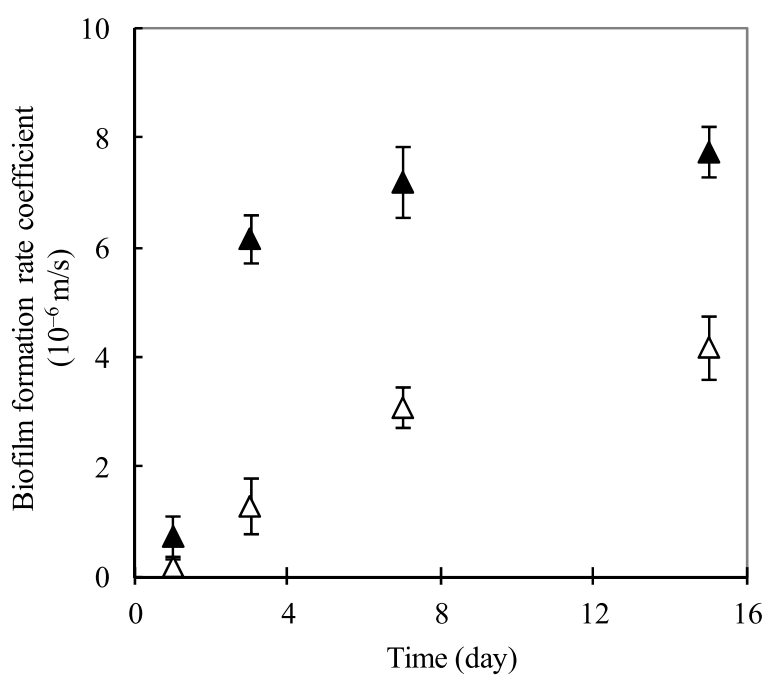

Fig. 3. Variations of biofilm formation rate coefficient as a function of incubation time ( $\Delta$, famine incubation run; $\Delta$, control run). Vertical bars represent the standard deviations calculated from triplicates.

tics, zeta potential, hydrophobicity and viscosity of biofilm biomass was carried out. To our knowledge, this is the first report in the literature on the bound EPS, protein and polysaccharide concentrations, surface charge, hydrophobicity profiles within a biofilm and the corresponding biofilm formation rate profile of the same biofilm. Table 1 shows a summary of the biofilm formation characteristics when the MABR culture was subjected to famine incubation under a low SLR. Bound EPS (e.g. bound protein) appeared to play a more important role in the formation of biofilm flocs than soluble EPS [22], and up to $91.6 \%$ of the total EPS was associated with bound EPS (Table 1). Famine incubation led to 2.0, 3.0, 3.5, 1.4, 2.2 and 1.5 times improvement in EPS, bound EPS, bound protein/bound polysaccharide ratio, hydrophobic, zeta potential and viscosity of physiochemical properties of biofilm biomass in contrast with the control run under the high SLR (see Tables 1 and 2). Fig. 4 shows the variation of biofilm formation rate coefficient as functions of different biofilm formation factors under famine incubation and control runs. We found that biofilm formation rate coefficient is variable as function of incubation time and increases with bound EPS content and hydrophobicity level (Fig. 4). For the famine incubation run, it is evident that bioflm formation rate significantly correlates with bound EPS content $\left(r_{\mathrm{p}}=0.954 ; p=0.046\right)$ as well as bound protein/bound polysaccharide ratio $\left(r_{\mathrm{p}}=0.956\right.$; $p=0.044$ ), indicating that it was a certain component of the EPS that dominated the biofilm formation rate coefficient, rather than total EPS (Table 3). Furthermore, hydrophobicity exhibited a moderate correlation with biofilm formation rate $\left(r_{\mathrm{p}}=0.921 ; p=0.079\right)$. However, zeta potential contributes negatively to the biofilm formation rate $\left(r_{\mathrm{p}}=-0.951 ; p=0.049\right)$ (Table 3$)$. It should be mentioned that EPS composition could affect hydrophobicity, viscosity and surface charge properties of biofilm biomass (Table 4). These findings are in agreement with previous studies which showed that the hydrophobicity and zeta potential of biomass were influenced by EPS component rather than total EPS quantity $[19,23]$. Bonet et al. [24] reported that the cell surface hydrophobicity is inversely correlated with the amount of polysaccharide composition, but positively with the protein proportion. It has also been reported that attached bacteria were much more hydrophobic than those in suspended sludge [25]. Lee et al. [26] stated that the protein in EPS had a strong positive influence on the hydrophobicity of microbial flocs, while polysaccharide had no remarkable influence. It was also observed that the protein in the EPS is mainly made up of hydrophobic amino acids [27]. Furthermore, protein appeared 
Table 1

Formation characteristics of membrane-aerated biofilm in the famine incubation run ${ }^{\mathrm{a}}$.

\begin{tabular}{|c|c|c|c|c|}
\hline \multirow[t]{2}{*}{ parameter } & \multicolumn{4}{|c|}{ incubation time (days) ${ }^{\mathrm{b}}$} \\
\hline & 1 & 3 & 7 & 15 \\
\hline EPS (mg/g-VSS) & $88.9 \pm 3.2$ & $103.9 \pm 4.0$ & $131.5 \pm 5.3$ & $149.2 \pm 5.2$ \\
\hline bound EPS (mg/g-VSS) & $46.3 \pm 1.7$ & $91.2 \pm 4.2$ & $129.6 \pm 5.1$ & $136.7 \pm 4.7$ \\
\hline bound protein/bound polysaccharide ratio & $1.6 \pm 0.05$ & $1.9 \pm 0.09$ & $2.1 \pm 0.1$ & $2.0 \pm 0.06$ \\
\hline hydrophobicity $\left({ }^{\circ}\right)$ & $32 \pm 1.1$ & $40 \pm 1.6$ & $50 \pm 2.7$ & $52 \pm 1.7$ \\
\hline zeta potential (mV) & $-16.8 \pm 0.53$ & $-10.5 \pm 0.41$ & $-5.1 \pm 0.26$ & $-3.7 \pm 0.09$ \\
\hline viscosity $(\mathrm{mPa} \cdot \mathrm{s})$ & $0.9 \pm 0.04$ & $1.3 \pm 0.05$ & $1.8 \pm 0.09$ & $2.1 \pm 0.04$ \\
\hline
\end{tabular}

a Grown on the gas-permeable membrane with famine incubation under a low SLR of $3.4 \mathrm{~g} / \mathrm{m}^{2} \mathrm{~d}$.

b The results are the means \pm standard deviations from triplicate experiments.

Table 2

Formation characteristics of membrane-aerated biofilm in the control run ${ }^{\mathrm{a}}$.

\begin{tabular}{|c|c|c|c|c|}
\hline \multirow[t]{2}{*}{ parameter } & \multicolumn{4}{|c|}{ incubation time (days) ${ }^{\mathrm{b}}$} \\
\hline & 1 & 3 & 7 & 15 \\
\hline EPS (mg/g-VSS) & $32.3 \pm 1.8$ & $43.2 \pm 2.4$ & $48.3 \pm 2.1$ & $107.8 \pm 4.7$ \\
\hline bound EPS (mg/g-VSS) & $18.1 \pm 0.9$ & $26.2 \pm 1.2$ & $28.6 \pm 1.6$ & $63.2 \pm 2.9$ \\
\hline bound protein/bound polysaccharide ratio & $0.4 \pm 0.03$ & $0.6 \pm 0.04$ & $0.5 \pm 0.02$ & $0.7 \pm 0.03$ \\
\hline hydrophobicity $\left(^{\circ}\right)$ & $29 \pm 1.5$ & $33 \pm 1.8$ & $31 \pm 1.7$ & $34 \pm 1.7$ \\
\hline zeta potential (mV) & $-26.1 \pm 1.1$ & $-16.7 \pm 0.7$ & $-19.4 \pm 1.2$ & $-16.2 \pm 0.9$ \\
\hline viscosity $(\mathrm{mPa} \cdot \mathrm{s})$ & $0.5 \pm 0.04$ & $0.9 \pm 0.05$ & $1.5 \pm 0.09$ & $1.1 \pm 0.04$ \\
\hline
\end{tabular}

a Grown on the gas-permeable membrane with control incubation under a high SLR of $12.8 \mathrm{~g} / \mathrm{m}^{2} \mathrm{~d}$.

b The results are the means \pm standard deviations from triplicate experiments.

Table 3

Pearson's correlation coefficient $\left(r_{p}\right)$ and $p$-values for linear correlations between biofilm formation rate coefficient and biomass properties.

\begin{tabular}{|c|c|c|c|c|}
\hline \multirow[t]{2}{*}{ adhesion factors of biofilm formation } & \multicolumn{2}{|c|}{ control run ${ }^{\mathrm{a}}$} & \multicolumn{2}{|c|}{ famine incubation run ${ }^{\mathrm{b}}$} \\
\hline & $r_{p}$ & $p$ & $r_{p}$ & $p$ \\
\hline EPS & 0.854 & 0.146 & 0.850 & 0.150 \\
\hline bound EPS & 0.861 & 0.139 & $0.954^{*}$ & 0.046 \\
\hline bound protein/bound polysaccharide ratio & 0.735 & 0.265 & $0.956^{*}$ & 0.044 \\
\hline hydrophobicity & 0.688 & 0.312 & 0.921 & 0.079 \\
\hline zeta potential & 0.710 & 0.290 & $-0.951^{*}$ & 0.049 \\
\hline viscosity & 0.778 & 0.222 & 0.894 & 0.106 \\
\hline
\end{tabular}

* Correlation is significant at the 0.05 level (2-tailed).

a Grown under a high SLR of $12.8 \mathrm{~g} / \mathrm{m}^{2} \mathrm{~d}$.

b Grown under a low SLR of $3.4 \mathrm{~g} / \mathrm{m}^{2} \mathrm{~d}$.

Table 4

Pearson's correlation coefficient $\left(\mathrm{r}_{\mathrm{p}}\right)$ and $p$-values for linear correlations between bound EPS and other factors.

\begin{tabular}{lll}
\hline adhesion factors of biofilm formation & \multicolumn{2}{l}{ bound EPS } \\
\cline { 2 - 3 } & $\mathrm{r}_{\mathrm{p}}$ & $p$ \\
\hline bound protein/bound polysaccharide ratio & $0.954^{*}$ & 0.046 \\
hydrophobicity & $0.995^{* *}$ & 0.005 \\
zeta potential & $-1.000^{* *}$ & 0.000 \\
viscosity & $0.978^{*}$ & 0.022 \\
\hline
\end{tabular}

* Correlation is significant at the 0.05 level (2-tailed)

** Correlation is significant at the 0.01 level (2-tailed).

to contribute significantly to the surface charge mainly due to ionization of functional groups of EPS in controlling surface charge of biofilm flocs.

\section{Discussion}

EPS have been recognized as a major constituent of biofilms and, according to the physicochemical properties (solubility and bindability), EPS can be classified into soluble and bound EPS [16]. The famine incubation triggered bacteria to secrete more EPS, especially more bound EPS (Tables 1 and 2). Since bound EPS are more hydrophobic and non-readily insoluble [22,28], they would facilitate the formation of biofilm flocs and their adhesion onto the membrane surface. EPS therefore serve as the backbone of the cohesive biofilm. An explanation for significant effect of hydrophobicity on biofilm formation is the hydrophobic interaction [29] between biofilm flocs and/or substratum. Higher hydrophobicity of biofilm flocs could present a stronger adherence of flocs with one another as well as onto the membrane surface for the driven force, thereby enhancing the biofilm formation. Results presented in Tables 3 and 4 indicate that the viscosity of the biofilm flocs had a close correlation with bound EPS and biofilm formation rate. The viscosity is a reflection of the magnitude of viscous substances, which may be considerably contributed by the polymers such as bound protein and polysaccharides. An increase of biopolymers in the biofilm flocs will increase its viscosity, hence enhance the biofilm formation rate. Thus the increase of the viscosity of the biofilm flocs was mainly caused by bound EPS.

In the present study, the zeta potential of microbial cells could be influenced by adsorbing the charged EPS. The presence of more proteins in the EPS contributes a large amount of positively charged amine groups to the EPS mixture. The fact that initiating microbial biofilm occurred with the reduction in surface charges of biofilm biomass indicates that microbial adsorption behavior can be approximately explained by electrostatic interaction based on the DLVO theory [30]. This theory, presented by Derjaguin, Landau, Verwey, and Overbeek, with an original aim of explaining colloid stability has been well accepted in depicting the adhesion behav- 

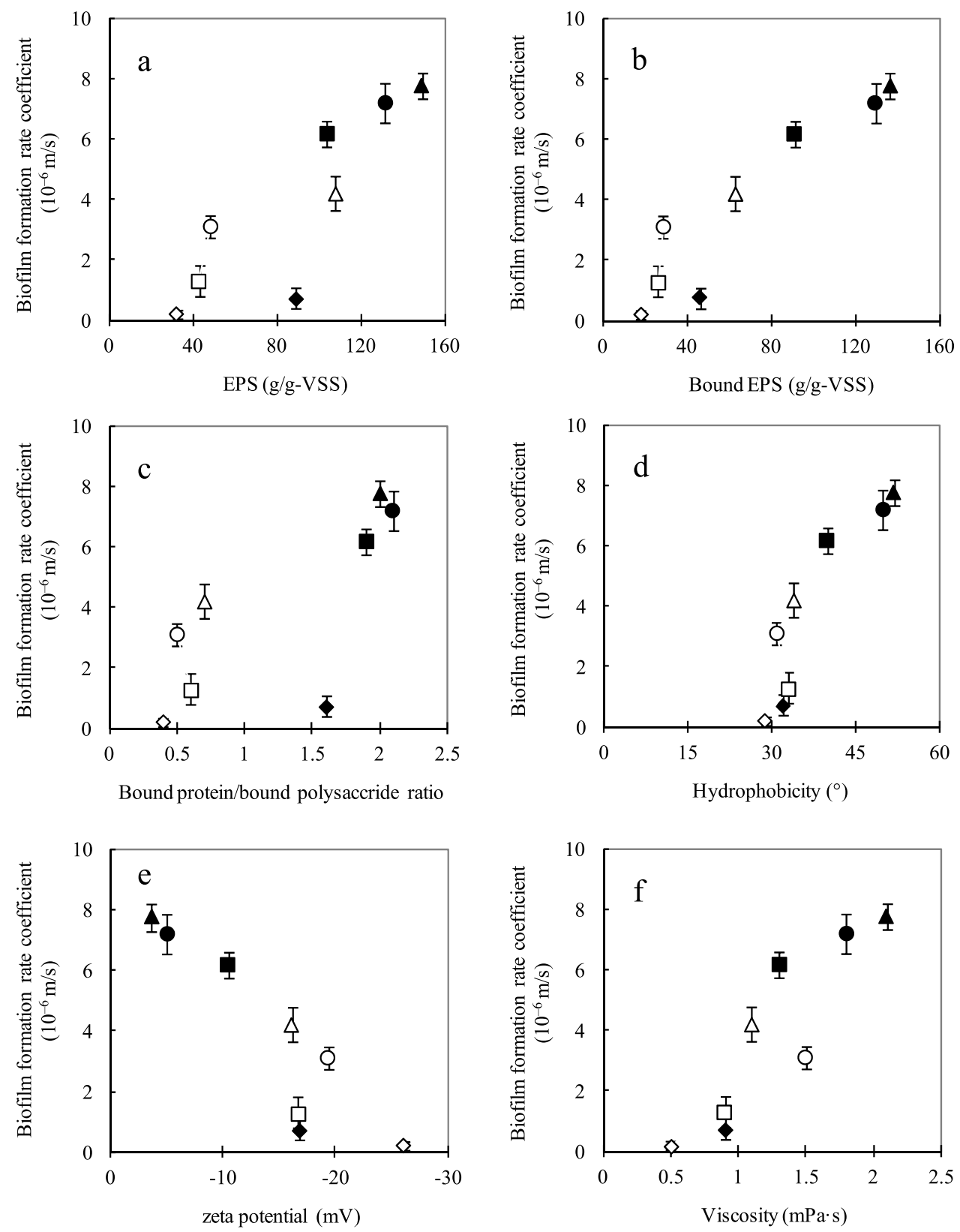

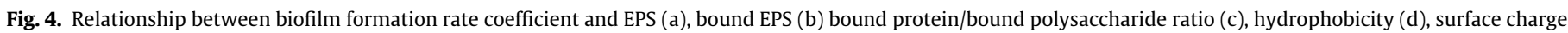

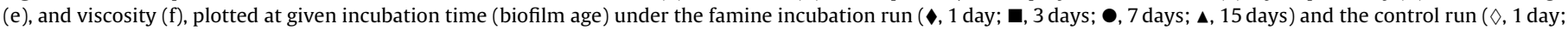
$\square$, 3 days; $\bigcirc, 7$ days; $\Delta, 15$ days). Vertical bars represent the standard deviations calculated from triplicates.

ior of microbial cells. The DLVO theory considers the overall Gibbs free energy as a function of the sum of attractive and repulsive forces over a distance between cells. Since bacteria have negatively charged surface under normal operation $\mathrm{pH}$ conditions, the electrostatic repulsion dominates or is balanced by a weak attraction so that microbial cells are located a certain distance from each other (secondary minimum). The increase in zeta potential of biofilm grown on the gas-permeable membrane with famine incubation could cut down the electrostatic repulsion between microbial cells or between microbial cells and membrane surface. Consequently, irreversible adhesion of microbial cells might occur with the reduction in electrostatic repulsion when bacterium reaches the primary minimum. Previous studies have reported that microbial aggregation could be initiated and accelerated via different approaches of altering bacterial surface charges. Reducing electrical repulsion between cells and solid surface could improve microbial adhesion performance [24].

Interestingly, the cell hydrophobicty appeared to be dependent on the change of cellular zeta potential. Previous studies have observed a strong inverse correlation between the surface charge and the contact angle of sludge [31], where activated sludge with more surface charges might absorb more EPS by electrostatic attraction which would increase the polar interactions of EPS with water molecules, and consequently increase the hydrophobic characteristics of sludge. In this study, however, famine incubation induced by a low SLR resulted in the increased bound EPS proportion and less charges of biofilm simultaneously. This indicated that the EPS quantity was not correlated positively with the surface charge.

Experimental results further indicated that famine incubation induced by low SLR influenced not only EPS concentration but 
also the specific EPS components and characteristics in biofilm. The high ratio of bound protein/bound polysaccharide and the positive correlation between biofilm formation rate and this ratio (Tables 1 and 3 ) suggest that the content and fraction of proteins in EPS significantly contributed to microbial biofilm formation. Due to the presence of amino groups in extracellular proteins and hydroxyl groups in extracellular polysaccharides, EPS can carry both positive and negative charges [32]. However, relatively fewer negative charges are carried by EPS with a high bound protein/bound polysaccharide ratio [19]. In this study, bound EPS represented about $85.3 \%$ total EPS content, indicating that bound proteins and bound polysaccharides were the dominant components in the EPS. Moreover the high bound protein/bound polysaccharide ratio suggests that charged EPS carried more positive charges and less negative charges. This resulted in the decrease of surface charges in biofilm biomass and an enhanced adsorption behavior of microbial cells and EPS, which in turn promoted formation of biofilm flocs. Cell hydrophobicity was also highly correlated with the protein/polysaccharide ratio [33]. Therefore, extracellular bound proteins increased hydrophobicity of cell surface, thus driving biofilm formation due to protein-dependent adhesion interaction.

\section{Conclusions}

A novel approach for initiating rapid formation of membraneaerated biofilm was demonstrated with an MABR. Applying famine incubation on the culture demonstrated an ability to enhance biofilm formation rate. Lowering the system surface loading rate could result in improvement in surface physicochemical properties of the culture favoring microbial biofilm formation. The improved bound EPS content, zeta potential, hydrophobicity and extracellular protein better facilitated biofilm formation. Our results suggest that microbial biofilm formation may be triggered by hydrophobic interaction, electrostatic attraction and protein-dependent adhesion induced by famine incubation. The strategy of facilitating biofilm formation can fully exploit the efficiency and scale-up of the emerging MABR technology.

\section{Acknowledgment}

This work was supported by the National Natural Science Foundation of China (Grant 51138009 and 50978246).

\section{References}

[1] R.M. Donlan, Biofilms: microbial life on surfaces, Emerg. Infect. Dis. 8 (2002) 881-890.

[2] C.S. Laspidou, L.A. Spyrou, N. Aravas, B.E. Rittmann, Material modeling of biofilm mechanical properties, Math. Biosci. 251 (2014) 11-15.

[3] M. Burmølle, J.S. Webb, D. Rao, L.H. Hansen, S.J. Sørensen, S. Kjelleberg, Enhanced biofilm formation and increased resistance to antimicrobial agents and bacterial invasion are caused by synergistic interactions in multispecies biofilms, Appl. Environ. Microbiol. 72 (2006) 3916-3923.

[4] C.A. Fux, J.W. Costerton, P.S. Stewart, P. Stoodley, Survival strategies of infectious biofilms, Trends Microbiol. 13 (2005) 34-40.

[5] S. Molin, T. Tolker-Nielsen, Gene transfer occurs with enhanced efficiency in biofilms and induces enhanced stabilization of the biofilm structure, Curr. Opin. Biotechnol. 14 (2003) 255-261.

[6] T.S. Weathers, C.P. Higgins, J.O. Sharp, Enhanced biofilm production by a toluene-degrading Rhodococcus observed after exposure to perfluoroalkyl acids, Environ. Sci. Technol. 49 (2015) 5458-5466.

[7] M. Herzberg, T.Z. Rezene, C. Ziemba, O. Gillor, K. Mathee, Impact of higher alginate expression on deposition of Pseudomonas aeruginosa in radial stagnation point flow and reverse osmosis systems, Environ. Sci. Technol. 43 (2009) 7376-7383.
[8] N. Dizge, D.Y. Koseoglu- Imer, A. Karagunduz, B. Keskinler, Effects of cationic polyelectrolyte on filterability and fouling reduction of submerged membrane bioreactor (MBR), J. Membr. Sci. 377 (2011) 175-181.

[9] T.A. Stenstrom, Bacterial hydrophobicity, an overall parameter for the measurement of adhesion potential to soil particles, Appl. Environ. Microbiol. 55 (1989) 142-147.

[10] A.E.J. van Merode, H.C. van der Mei, H.J. Busscher, B.P. Krom, Influence of culture heterogeneity in cell surface charge on adhesion and biofilm formation by Enterococcus faecalis, J. Bacteriol. 188 (2006) 2421-2426.

[11] E. Casey, B. Glennon, G. Hamer, Review of membrane aerated biofilm reactors, Resour. Conserv. Recycl. 27 (1999) 203-215.

[12] E. Syron, E. Casey, Membrane-aerated biofilms for high rate biotreatment: performance appraisal, engineering principles, scale-up, and development requirements, Environ. Sci. Technol. 42 (2008) 1833-1844.

[13] Y. Zhang, F. Wang, X. Zhu, J. Zeng, Q. Zhao, X. Jiang, Extracellular polymeric substances govern the development of biofilm and mass transfer of polycyclic aromatic hydrocarbons for improved biodegradation, Bioresour. Technol. 193 (2015) 274-280

[14] D. Hadjiev, D. Dimitrov, M. Martinov, O. Sire, Enhancement of the biofilm formation on polymeric supports by surface conditioning, Enzyme Microb. Technol. 40 (2007) 840-848.

[15] R.Y. Zhang, S. Bellenberg, L. Castro, T.R. Neu, W. Sand, M. Vera, Colonization and biofilm formation of the extremely acidophilic archaeon Ferroplasma acidiphilum, Hydrometallurgy 150 (2014) 245-252.

[16] T.G. Li, R.B. Bai, J.X. Liu, Distribution and composition of extracellular polymeric substances in membrane-aerated biofilm, J. Biotechnol. 135 (2008) 52-57.

[17] APHA, Standard Methods for the Examination of Water and Wastewater, 20th ed., American Public Health Association, Washington DC, 1998.

[18] T.G. Li, J.X. Liu, R.B. Bai, F.S. Wong, Membrane-aerated biofilm reactor for the treatment of acetonitrile wastewater, Environ. Sci. Technol. 42 (2008) 2099-2104.

[19] B.Q. Liao, D.G. Allen, I.G. Droppo, G.G. Leppard, S.N. Liss, Surface properties of sludge and their role in bioflocculation and settleability, Water Res. 35 (2001) 339-350.

[20] M.J. Dubois, K.A. Gills, J.K. Hamilton, P.A. Reber, F. Smith, Colorimetric method for determination of sugars and related substances, Anal. Chem. 28 (1956) 350-356.

[21] M.M. Bradford, A rapid and sensitive method for the quantification of microgram quantities of protein utilizing the principle of protein-dye binding, Anal. Biochem. 72 (1976) 248-254.

[22] C.S. Laspidou, B.E. Rittmann, A unified theory for extracellular polymeric substances, soluble microbial products, and active and inert biomass, Water Res. 36 (2002) 2711-2720.

[23] Y.F. Dufrêne, C.J.P. Boonaert, P.G. Rouxhet, Adhesion of Azospirillum brasilense: role of proteins at the cell-support interface, Colloids Surf. B 7 (1996) $113-128$.

[24] R. Bonet, M. Simon-Pujol, F. Congregado, Effects of nutrients on exopolysaccharide production and surface properties of Aeromonas salmonicida, Appl. Environ. Microbiol. 59 (1993) 2437-2441.

[25] R. Dewanti, A.C.L. Wong, Influence of culture conditions on biofilm formation by Escherishia Coli 0157: H7, Int. J. Food Microbiol. 26 (1995) 147-164.

[26] J. Lee, W.Y. Ahn, C.H. Lee, Comparison of the filtration characteristics between attached and suspended growth microorganisms in submerged membrane bioreactor, Water Res. 35 (2011) 2435-2445.

[27] F.J. Jorand, F.P. Boue-Bigne, J.C. Block, V. Urbain, Hydrophobic/hydrophilic properties of activated sludge exopolymeric substances, Water Sci. Technol. 37 (1998) 307-315.

[28] C.N. Hirst, H. Cyr, I.A. Jordan, Distribution of exopolymeric substances in the littoral sediments of an oligotrophic lake, Microb. Ecol. 46 (2003) 23-32.

[29] M.K.H. Liew, A.G. Fane, P. Rogers, Hydraulic resistance and fouling of microfilters by Candida utilis in fermentation broth, Bioeng. Biotechnol. 48 (1995) 108-117.

[30] P.G. Rouxhet, N. Mozes, Physical chemistry of the interaction between attached microorganisms and their support, Water Sci. Technol. 22 (1990) $1-16$.

[31] D.T. Sponza, Investigation of extracellular polymer substances (EPS) and physicochemical properties of different activated sludge flocs under steady-state conditions, Enzyme Microb. Technol. 32 (2003) 375-385.

[32] P.V. Bramhachari, S.K. Dubey, Isolation and characterization of exopolysaccharide produced by Vibrio harveyi strain VB23, Lett. Appl. Microbiol. 43 (2006) 571-577.

[33] S. Kang, H. Choi, Effect of surface hydrophobicity on the adhesion of $S$. cerevisiae onto modified surfaces by poly(styrene-ran-sulfonic acid) random copolymers, Colloids Surf. B Biointerfaces 46 (2005) 70-77. 